\author{
Heinrich MeYbohm* - Adriano ZanetTi ${ }^{* *}$
}

\title{
Die Arten der Gattung Cephennium Müller \& Kunze, 1822 (Coleoptera, Staphylinidae, Scydmaeninae) aus der Provinz Verona (Italien)
}

\begin{abstract}
Riassunto: Le specie del genere Cephennium Müller \& Kunze, 1822 (Coleoptera, Staphylinidae, Scydmaeninae) della provincia di Verona.

Cinque specie del genere Cephennium vengono segnalate per la Provincia di Verona. Quattro specie appartengono al sottogenere Cephennium s.str.: Cephennium (Cephennium) jureceki Machulka, 1930; Cephennium (Cephennium) rossii Castellini, 2011; Cephennium (Cephennium) lessinicum nov. spec.; Cephennium (Cephennium) settei nov. spec.; una specie appartiene al sottogenere Neocephennium Apfelbeck, 1911: Cephennium (Neocephennium) punctatum nov. spec.. Le nuove specie vengono descritte e vengono fornite illustrazioni di tutte le cinque specie. Viene fornita una tavola per distinguere le quattro specie del sottogeneri Cephennium s. str.
\end{abstract}

Abstract: The species of the genus Cephennium Müller \& Kunze, 1822 (Coleoptera, Staphylinidae, Scydmaeninae) in the province of Verona (Italy).

Five species of the genus Cephennium are recorded from the North Italian province Verona. Four species belong to the subgenus Cephennium s.str:: Cephennium (Cephennium) jureceki Machulka, 1930; Cephennium (Cephennium) rossii Castellini, 2011; Cephennium (Cephennium) lessinicum nov. spec.; Cephennium (Cephennium) settei nov. spec.. One species belongs to the subgenus Neocephennium Apfelbeck, 1911: Cephennium (Neocephennium) punctatum nov. spec.. The new species are described and illustrations are given to all five species. An identification table for the four species of the subgenus Cephennium s.str. is provided.

Key words: Coleoptera, Staphylinidae, Scydmaeninae, Cephennium, New species, Northern Italy.

\section{EINLEITUNG}

Der Publikation liegt umfangreiches Material von Scydmaeninen aus der norditalienischen Provinz Verona zu Grunde. Ganz überwiegend stammt es aus umfangreichen koleopterologischen Aufsammlungen von Alberto Sette, die er in der gesamten Provinz und vor allem im Bereich des "Parco Naturale Regionale della Lessinia" durchgeführt hat, um einen Katalog der Käfer zu erstellen, die in dieser Provinz leben.

Die Bereitschaft des Erstautors, die Scydmaeninen zu determinieren, war von Anfang an damit verbunden, entweder Manuskriptnamen von Claude Besuchet zu verwenden oder einige Arten der Gattung Cephennium neu zu beschreiben. Claude Besuchet hat Anfang der 1960iger Jahre umfangreiche Studien der Gattung durchgeführt, von fast allen beschriebenen und von vielen nicht beschriebenen Arten detailgetreue Zeichnungen der Aedeagi angefertigt und den unbeschriebenen Arten Manuskriptnamen gegeben. Leider ist es nicht zu einer Publikation seiner Ergeb- nisse gekommen. Aus verschiedenen Gründen werden die von ihm gewählten Namen bei den nun erfolgten Beschreibungen nicht übernommen. Alle Holotypen und Paratypen befinden sich im Museo Civico di Storia Naturale di Verona.

Die Koordinaten der Fundstellen sind auf den Etiketten der Individuen nicht verzeichnet. Die Zahlen und Ortsbezeichnungen in eckigen Klammern korrigieren Höhenangaben und manchmal Fundortbezeichnungen auf den Etiketten.

ABKÜRZung. Aed. Präp. mindestens ein Exemplar mit Aedeagus-Präparat.

\section{Arten des Subgenus Cephennium Müller \& Kunze, 1822}

Cephennium (Cephennium) lessinicum nov. spec. =Cephennium (Cephennium) pechlaneri Besuchet i.l.

Diagnose. Von nach äußeren Merkmalen kaum zu

*Heinrich Meybohm, Wassenkamp 2a, 22927 Großhansdorf, Deutschland. E-mail: hmeybohm@t-online.de

**Adriano Zanetti, Museo Civico di Storia Naturale, Lung. P.ta Vittoria 9, 37129 Verona, Italy. E-mail: zanet@easyasp.it 
trennenden verwandten Arten des Subgenus vor allem durch Merkmale des Aedeagus verschieden.

Holotypus. đ Parco Lessinia (VR) Val Fraselle mt. 900

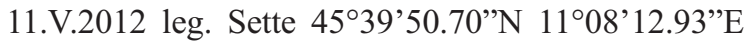
(Museo Civico di Storia Naturale di Verona).

Paratypen. Parco Naturale Regionale della Lessinia (Verona): Camposilvano mt 1200 45³7'57.79”N

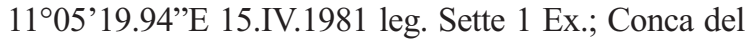

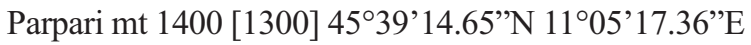
13.IV.2010 leg Sette 1 Ex.; Giazza Val Fraselle (VR)

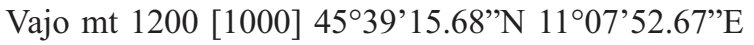
1.V.1975 leg. Sette 1 Ex.; S. Giorgio mt 1500 [Vallone del Malera mt 1600] 45 $41^{\prime} 14.97^{\prime \prime} \mathrm{N} 11^{\circ} 05^{\prime} 56.56^{\prime \prime} \mathrm{E}$ 25.V.1978 leg. Sette 3 Ex.; Vaio dei Falconi (VR) mt 500 [600] 45³6'37.74”'N 1058'32.36”'E 6.V.1975 leg. Sette 1 Ex.; Vajo di Fumane 27.3.1991 leg. Zanetti 1 Ex.; Val Fraselle - Alar mt $1000 \quad 45^{\circ} 39^{\prime} 33.45^{\prime \prime} \mathrm{N}$

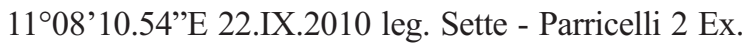
Aed. Präp.; Val Fraselle mt 1000 Alar 4539'33.45'N

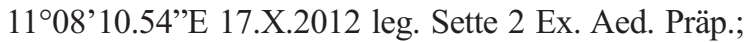
Val Fraselle mt $90045^{\circ} 39^{\prime} 50.70^{\prime \prime} \mathrm{N} 11^{\circ} 08^{\prime} 12.93^{\prime \prime} \mathrm{E}$ 11.V.2012 leg. Sette 1 Ex. Aed. Präp.; Val Fraselle mt $90045^{\circ} 39^{\prime} 50.70^{\prime \prime} \mathrm{N} 11^{\circ} 08^{\prime} 12.93$ 'E 6.IV.2012 leg. Sette - Parricelli 2 Ex. Aed. Präp.; Val Fraselle mt 900 vaglio sotto conifera $45^{\circ} 39^{\prime} 50.70^{\prime} \mathrm{N} \quad 1^{\circ} 08^{\prime} 12.93^{\prime \prime} \mathrm{E}$ 2.XII.2011 leg. Sette - Parricelli 1 Ex.; Val Revolto mt 900 primo tornante - Rive del progno Lavaggio del ter-

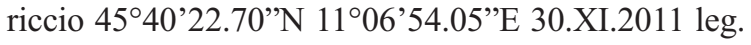
Sette 4 Ex. Aed. Präp.; Val Squaranto mt 1000-1100

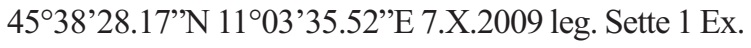
Außerhalb Parco della Lessinia: Progno di Mezzane

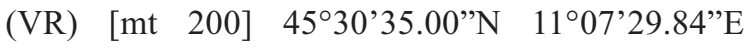
10.IV.1983 leg Sette 2 Ex. Aed. Präp.; Roveré (VR) S. Rocco - Vajo mt 500 - Castagneto $45^{\circ} 33^{\prime} 44.47^{\prime \prime} \mathrm{N}$ $11^{\circ} 05^{\prime} 35.59^{\prime}$ E 2.IV.2010 leg. Sette 5 Ex.; Roveré (VR) S. Rocco Loc. Porcara $\mathrm{mt} 800$ [700] $45^{\circ} 34^{\prime} 21.38^{\prime \prime} \mathrm{N} 11^{\circ} 04^{\prime} 50.16^{\prime \prime} \mathrm{E}$ 1.III.2010 leg. Sette 1 Ex.; Roveré (VR) San Rocco - Vaio mt 500

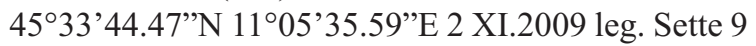
Ex. Aed. Präp.; Val Tramigna (VR) Loc. Sengelle [Cengelle] mt $30045^{\circ} 30^{\prime} 03.19^{\prime \prime} \mathrm{N} 11^{\circ} 11^{\prime} 52.19^{\prime \prime} \mathrm{E}$ 5.XI.2009 1 Ex., 9.I.2010 2 Ex. Aed. Präp.

BESCHREIBUNG. Körperlänge 0,92-1,02 mm. Einfarbig rotbraun gefärbt.

Fühler mit 3-gliedriger Keule. Glieder VI und VIII kleiner als die benachbarten, II-VI walzenförmig, der Länge nach wenig gerundet, $\mathrm{V}$ mehr oder weniger deutlich breiter und länger als III und IV, die kaum verschieden sind, VII und VIII kurzoval, der Länge nach stärker gerundet, IX und X krugförmig, XI langoval.

Länge: Breite der Fühlerglieder II 9:6, III 6:5, IV 6:5, V 6:5,5, VI 5:5, VII 6,5:6, VIII 5:6, IX 9:9,5, X 9:11, XI 18:11

Pronotum: Etwa 1,2 mal so breit wie lang. GröBte Breite des Pronotums 0,40-0,44 mm, sie ist $1 / 3$ der Länge des Pronotums vom Vorderrand entfernt. Seitenränder nach vorn gleichmäßig gerundet stark konvergierend, nach hinten geradlinig schwach konvergierend. Hinterwinkel schwach stumpfwinklig, nicht abgesetzt. Mediane Beule vor dem Hinterrand deutlich aber schwach entwickelt.

Protibia beim $\widehat{\delta}$ im apikalen Drittel nur sehr schwach eingebuchtet (Abb. 1C).

Elytren: Etwa 1,38 mal so lang wie zusammen breit. Geringfügig schlanker als bei $C$. jureceki. Am Hinterende kaum abgestutzt, weniger als bei $C$. jureceki und C. rossii. Basale Pore von feinen Setae radial überdeckt.

Metasternum überall gleichmäßig fein weitläufig punktiert. Kielchen des Metasternalfortsatzes nicht nach hinten verlängert.

ô: Metasternum mit schwachem Eindruck. Aedeagus (Abb. 1A,B).

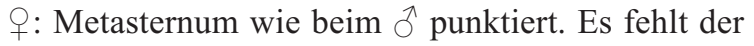
mediane Eindruck.

Derivatio NOminis. Benannt nach den Monti Lessini in der Provinz Verona.

Vergleiche. Siehe Bestimmungstabelle.

Bionomie. Die Exemplare wurden aus Streu in Misch-, Nadel-, Buchen-, Kastanienwäldern, unter Pinus mugo und an Flussufern, auf kalkreichem Boden, in Höhenlagen zwischen 200 und 1600 m gesammelt.

Cephennium (Cephennium) settei nov. spec.

$=$ Cephennium (Cephennium) brixianum Besuchet i.l.

DiAgNoSE. Von nach äußeren Merkmalen kaum zu trennenden verwandten Arten des Subgenus vor allem durch Merkmale des Aedeagus verschieden.

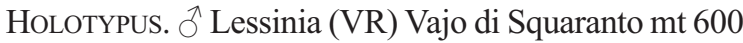

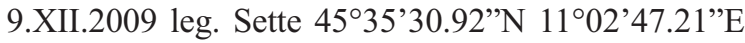
(Museo Civico di Storia Naturale di Verona) 
Paratypen. Parco Naturale Regionale della Lessinia (VR): Fittanze mt 1300 [1350] 4541'00.61'N $10^{\circ} 59^{\prime} 13.29^{\prime \prime} \mathrm{E} 16 . \mathrm{V} .1976$ leg. Sette 2 Ex.; Fittanze mt $135045^{\circ} 41^{\prime} 00.61$ 'N 1059'13.29”'E 26.X.2009 leg. Sette 2 Ex.; Vajo del Falconi mt 550 4536'37.74'N 1058'32.36”'E 23.III.2011 leg. Sette - Parricelli 1 Ex.; Vajo dell'Anguilla Vallina mt 1200-

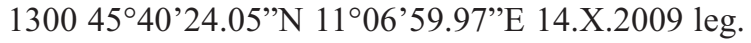
Sette 2 Ex.; Vajo della Marchiora (VR) mt 400 [550] 13.I.2010 leg. Sette 3 Ex.; Vajo della Marchiora mt 450 [550] 45³6'39.11'”N 1058'18.00’'E 25.XI.2009 leg. Sette 20 Ex.; Val di Squaranto mt 1100

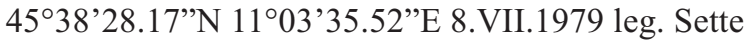
6 Ex.; Val Fraselle - Alar mt 1000 45³9'33.45”N $11^{\circ} 08^{\prime} 10.54^{\prime \prime}$ 2 22.IX.2010 leg. Sette - Parricelli 1 Ex.; Val Revolto faggeta mt $110045^{\circ} 41^{\prime} 25.74^{\prime \prime} \mathrm{N}$ $11^{\circ} 07^{\prime} 13.53$ ”E 23.X.2009 leg. Sette 1 Ex. Val Revolto $\mathrm{mt} 1000 \quad 45^{\circ} 40^{\prime} 24.05^{\prime \prime} \quad \mathrm{N} 11^{\circ} 06^{\prime} 59.977^{\prime} \mathrm{E}$ 23.VII.2012 leg. Sette 7 Ex. Aed. Präp.; Val Revolto mt $105045^{\circ} 40^{\prime} 56.02$ " N11 $07^{\circ}$ '01.57”'E 14.XI.2011 leg. Sette 1 Ex.; Val Revolto mt 900 Primo tornante Rive del progno Lavaggio del terriccio

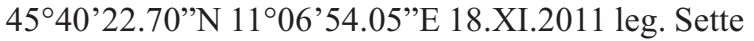

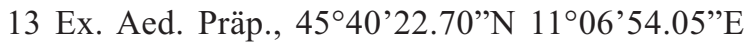
30.XI.2011 leg. Sette 1 Ex.; Val Squaranto mt 10001100 4538'28.17'N 11'03'35.52"'E 7.X.2009 leg. Sette 3 Ex.

Außerhalb Parco della Lessinia: Castagné (VR)

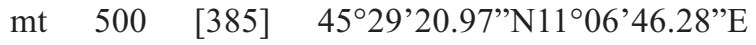
5.VI.1977 leg. Sette 8 Ex.; Mezzane (VR) Vaio mt 300

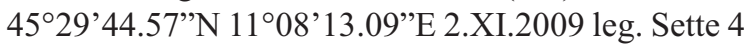
Ex. Aed. Präp.; Monte Baldo (VR) Val Trovaj mt 1300

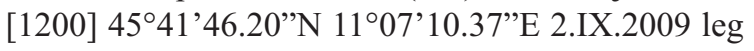
Sette 4 Ex.; Negrar (VR) Montecchio mt 500 4531'08.04'N 1058'53.47'”E 22.I.2010 leg. Sette 5 Ex. Aed. Präp.; Progno di Mezzane (VR) mt 250

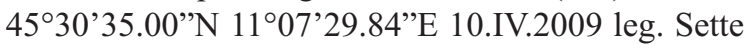
1 Ex. Aed. Präp.; Roveré (VR) S. Rocco - Vajo mt 500 - Castagneto $45^{\circ} 33^{\prime} 44.47^{\prime \prime} \mathrm{N} \quad 1^{\circ} 05^{\prime} 35.59^{\prime \prime} \mathrm{E}$ 2.IV.2010 leg. Sette 2 Ex.; Roveré (VR) S. Rocco Loc. Porcara mt [700] 45 $34^{\prime} 21.38^{\prime \prime} \mathrm{N} 11^{\circ} 04^{\prime} 50.16^{\prime \prime} \mathrm{E} 1$ III.2010 leg. Sette 1 Ex.; S. Anna d'Alfaedo (VR) Ceredo $\mathrm{mt} 750 \quad 45^{\circ} 37^{\prime} 00.86^{\prime \prime}$ N10 $58^{\prime} 25.23$ ” E 19.III.2012 leg. Sette - De Togni 4 Ex. Aed. Präp.; Tregnago (VR) Loc. Battistini mt $50045^{\circ} 32$ '26.62”'N $11^{\circ} 08^{\prime} 43.29^{\prime \prime} \mathrm{E}$ 8.II.2010 leg. Sette 1 Ex.; Tregnago (VR) Loc. La Collina mt 650 [750] 45 38’33.59”N $11^{\circ} 11^{\prime} 02.23$ 'E 2.IV.2010 leg. Sette 2 Ex.; Tregnano (VR) M.te Bellocca mt $750 \quad 45^{\circ} 32^{\prime} 19.59^{\prime \prime} \mathrm{N}$

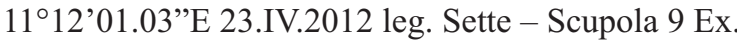
Aed. Präp., 7.V.2012 leg. Sette 1 Ex.; Vajo di Squaranto (VR) $\mathrm{mt} 550 \quad 45^{\circ} 35^{\prime} 30.92^{\prime \prime} \mathrm{N}$ $11^{\circ} 02^{\prime} 47.21^{\prime \prime}$ 20.IV.1975 leg. Sette 1 Ex. ; Vajo di Squaranto (VR) $\mathrm{mt} \quad 600 \quad 45^{\circ} 35^{\prime} 30.92^{\prime \prime} \mathrm{N}$ $11^{\circ} 02^{\prime} 47.21$ 'E 9.XII.2009 leg. Sette 5 Ex.; Val Tramigna (VR) Tregnago loc. Finetti mt 500 4532'18.62”N 11¹1'53.21”E 16.V.2009 leg. Sette 1 Ex.; Val Tramigna (VR) loc. Campiano mt 450 [loc.

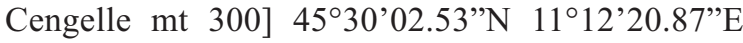
9.V.1976 leg. Sette 1 Ex.; Val Tramigna (VR) Loc. Cengelle mt $25045^{\circ} 30^{\prime} 03.19^{\prime \prime} \mathrm{N} 11^{\circ} 11^{\prime} 52.19^{\prime \prime} \mathrm{E}$ 5.XI.2009 leg. Sette 3 Ex.; Val Tramigna (VR) Loc. Sengelle [Cengelle] $\mathrm{mt} 300 \quad 45^{\circ} 30^{\prime} 02.53^{\prime \prime} \mathrm{N}$ $11^{\circ} 12^{\prime} 20.87^{\prime \prime} E$ 19.I.2010 leg. Sette 1 Ex.; Val Trami-

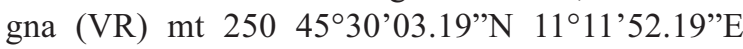
6.IV.2009 leg. Sette 2 Ex.

BeSCHREIBUNG. Körperlänge 1,07-1,24 mm. Einfarbig rotbraun gefärbt.

Fühler mit 3-gliedriger Keule. Glieder: VI und VIII kleiner als die benachbarten, II-VI walzenförmig, der Länge nach wenig gerundet, $\mathrm{V}$ mehr oder weniger deutlich breiter und länger als III und IV, die kaum verschieden sind, VII und VIII kurzoval, der Länge nach stärker gerundet, IX und X krugförmig, XI langoval.

Länge : Breite der Fühlerglieder II 10:7, III 7:6, IV 7:6, V 8:6, VI 6:6, VII 8:7, VIII 6:7, IX 10:11, X $11: 11,5$, XI 20:12

Pronotum: Etwa 1,2 mal so breit wie lang. GröBte Breite des Pronotums 0,46-0,50 mm, sie ist $1 / 3$ der Halsschildlänge vom Vorderrand entfernt. Seitenränder nach vorn gleichmäßig gerundet stark konvergierend, nach hinten geradlinig schwach konvergierend. Hinterwinkel schwach stumpfwinklig, nicht abgesetzt. Mediane Beule vor dem Hinterrand deutlich und kräftig entwickelt.

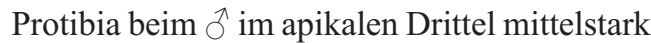
eingebuchtet (Abb. 1F).

Elytren: Etwa 1,38 mal so lang wie zusammen breit. Geringfügig schlanker als bei $C$. jureceki. Am Hinterende kaum abgestutzt, weniger als bei $C$. jureceki und C. rossii. Basale Pore von feinen Setae radial überdeckt.

Kielchen des Metasternalfortsatzes nach hinten jeweils in eine feine Kante verlängert, die maximal bis zur Mitte des Metasternums reicht.

$\delta$ : Metasternum median breit muldenförmig tief eingedrückt. Eindruck weitläufig fein punktiert, wulstige 


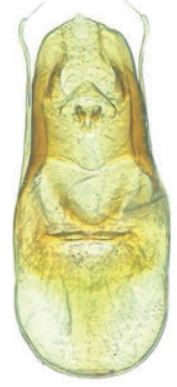

A

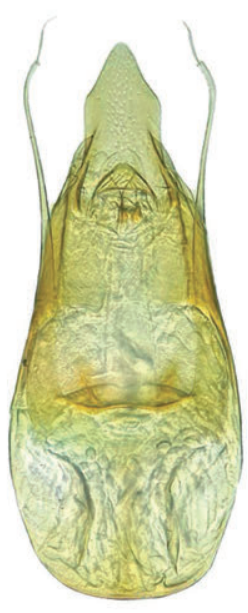

G

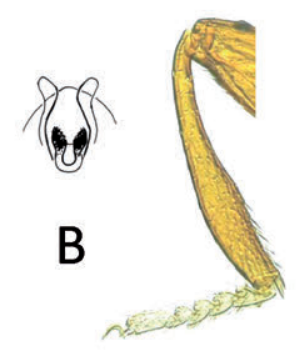

C

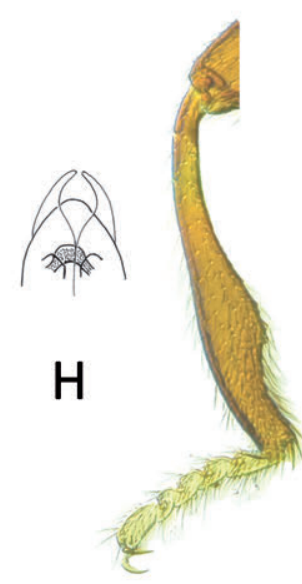

I

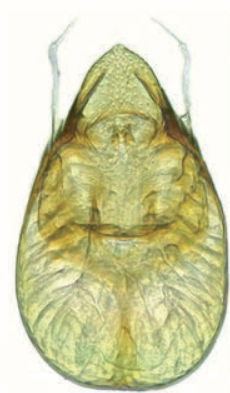

D
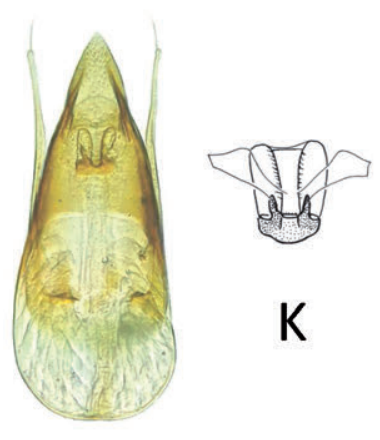

K

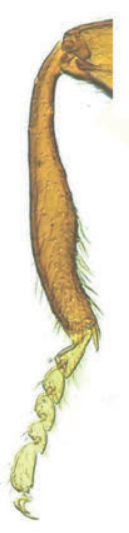

J

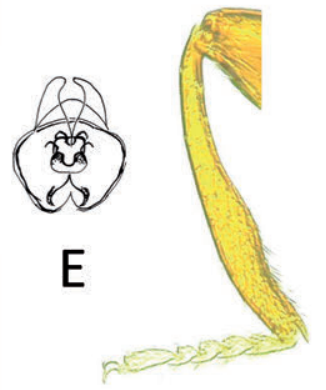

$\mathrm{F}$

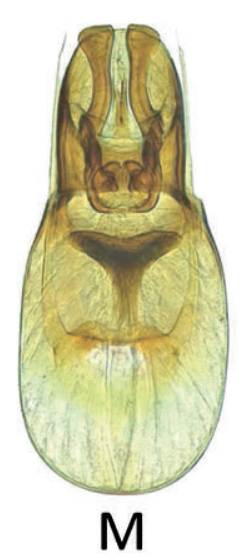

$00 \mu \mathrm{m}(\mathrm{B}, \mathrm{E}, \mathrm{H}, \mathrm{K})$

$100 \mu \mathrm{m}(\mathrm{A}, \mathrm{C}, \mathrm{D}, \mathrm{F}, \mathrm{G}, \mathrm{I}, \mathrm{J}, \mathrm{L}, \mathrm{M})$

Abb. 1. A-C. Cephennium (Cephennium) lessinicum nov. spec.: A - Aedeagus (Val Fraselle mt 900); B - ausstülpbare Sklerite des Aedeagus (Holotypus); C - Protibia ồ (San Rocco - Vaio mt 500). D-F. Cephennium (Cephennium) settei nov. spec.: D - Aedeagus (Val Revolto mt 1000); E - ausstülpbare Sklerite des Aedeagus (Holotypus); F - Protibia $\widehat{\widehat{O}}$ (Vajo di Squaranto mt 600). G-I. Cephennium (Cephennium) jureceki Machulka, 1930: G - Aedeagus (Val Fraselle mt 900); H - ausstülpbare Sklerite des Aedeagus (Mte Bellocca mt 750); I - Protibia ô (Val Fraselle mt 900). J-L. Cephennium (Cephennium) rossii Castellini, 2011. J - Aedeagus (Valle di Fumane Mulin de Cao mt 300); K - ausstülpbare Sklerite des Aedeagus (Vallone del Malera mt 1400); L - Protibia 0 (Cima Bella Lasta mt 1750). M. Cephennium (Neocephennium) punctatum nov. spec. Aedeagus (Val Fraselle mt 900). 
Begrenzung des Eindrucks dichter grob punktiert, die grobe Punktierung erstreckt sich mehr oder weniger weit auf die Seiten. Aedeagus (Abb. 1D,E).

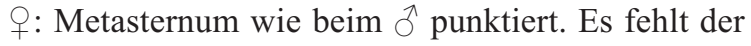
mediane Eindruck.

Derivatio nOminis. Benannt nach Alberto Sette, dem Sammler des umfangreichen Materials.

Vergleiche. Siehe Bestimmungstabelle.

Bionomie. Die Exemplare wurden aus Streu von Misch-, Nieder-, Nadel-, Kastanien- und Buchen-Wäldern, an Bachufern, auf kalkreichem Boden, oft im Tal, in Höhenlagen zwischen 250 und 1350 m gesammelt.

\section{Cephennium (Cephennium) jureceki Machulka, 1930}

Protibia beim ${ }^{\wedge} \mathrm{im}$ apikalen Drittel stark eingebuchtet (Abb. 1I).

Aedeagus (Abb. 1G,H)

Die Art ist in den Nord-Italienischen Regionen Veneto, Lombardia und Trento weit verbreitet. Zahlreiche Belege aus der Provinz Verona sind im vorgelegten Material enthalten. Sie stammen sowohl aus dem Parco Lessinia wie auch von Fundstellen außerhalb des Parco Lessinia.

Cephennium (Cephennium) rossii Castellini, 2011 $=$ Cephennium (Cephennium) ambiguum Besuchet i.l.

Protibia beim ${ }^{\Uparrow}$ im apikalen Drittel mittelstark eingebuchtet (Abb. 1L).

Aedeagus (Abb. 1J,K).

Castellini beschreibt die Art nach 5 Exemplaren aus der Region Lazio. Als Körperlänge gibt er 1,17-1,24 mm an. Sechs Exemplare aus Umbrien (leg. Wunderle, coll. Meybohm) sind 1,14-1,15 mm lang. Dagegen sind alle unten aufgelisteten 12 Exemplare aus der Provinz Verona wie auch alle 38 Exemplare in der Sammlung Meybohm aus der Region Trento deutlich kleiner und nur 1,00-1,10 mm lang. Weitere Unterschiede waren nicht erkennbar, woraus sich auch die Übereinstimmung mit Cephennium (Cephennium) ambiguum Besuchet i.l. ergibt.

Parco Naturale Regionale della Lessinia (VR): Cima Bella Lasta Mugheto mt 1750 4541'26.38'N $11^{\circ} 06^{\prime} 16.95^{\prime \prime}$ E 26.V.2010 leg. Sette 1 Ex. Aed. Präp.; Corno d'Aquilio mt $1450 \quad 45^{\circ} 40^{\prime} 29.56^{\prime \prime} \mathrm{N}$
1057’06.36'E 14.IV.2011 leg. Sette - Parricelli 3 Ex. Aed. Präp.; Rif. Revolto mt $130045^{\circ} 41^{\prime} 45.20^{\prime \prime} \mathrm{N}$ $11^{\circ} 07^{\prime} 10.37^{\prime \prime} E$ 1.X. 2012 leg. Sette 1 Ex. Aed. Präp.; Vajo dell'Anguilla Vallina $\mathrm{mt}$ 1200-1300 4540'24.53”N 1101'30.98”E 14.X.2009 leg. Sette 1 Ex. Aed. Präp.; Vajo di Squaranto mt 1000-1100

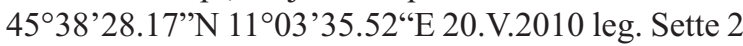
Ex. Aed. Präp.; Val Revolto mt 900 Primo tornante Rive del progno Lavaggio del terriccio $45^{\circ} 40^{\prime} 22.78^{\prime} \mathrm{N}$ $11^{\circ} 06^{\prime} 54.05^{\prime}$ E 18.XI.2011 leg. Sette 1 Ex.; Valle di Fumane Mulin de Cao mt 300 45'35'25.86" N1054’26.46”'E 25.II.2011 leg. Sette - Parricelli 2 Ex. Aed. Präp.; Vallone del Malera mt 1400 [1600] 16.IX.2010 4541'14.97’N1105'56.56”'E leg. Sette Parricelli 1 Ex. Aed. Präp.

\section{Art des Subgenus Neocephennium Apfelbeck, 1911}

Cephennium (Neocephennium) punctatum nov. spec. $=$ Cephennium (Neocephennium) baldense Besuchet i.l.

DiAGNOSIS. Von nach äußeren Merkmalen kaum zu trennenden verwandten Arten des Subgenus vor allem durch die Gestalt der ausstülpbaren Sklerite des Aedeagus verschieden.

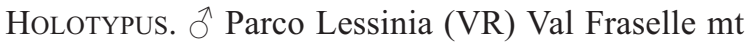

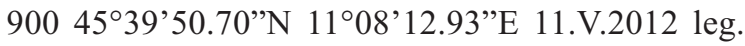
Sette (Museo Civico di Storia Naturale di Verona) PARATYPEn. Parco Naturale Regionale della Lessinia (VR): Cima Bella Lasta Mugheto mt 1750

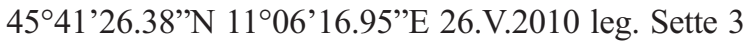
Ex. Aed. Präp.; Conca del Parpari mt 1400 [1300] $45^{\circ} 39^{\prime} 14.65^{\prime}{ }^{\prime} N 1^{\circ} 05^{\prime} 17.36^{\prime}$ 'E 13.IV.2010 leg. Sette 7 Ex; Rif. Revolto $\mathrm{mt} 1300 \quad 45^{\circ} 41^{\prime} 45.20^{\prime \prime} \mathrm{N}$

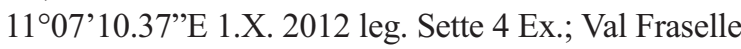
mt 900 4539'50.70”'N 1108'12.93”'E 11.V.2012 leg. Sette 22 Ex. Aed. Präp.; Val Fraselle mt 900 $45^{\circ} 39^{\prime} 50.70^{\prime \prime} \mathrm{N} 11^{\circ} 08^{\prime} 12.93^{\prime}$ 'E 31.III.2011 leg. Sette Parricelli 12 Ex.; Val Fraselle mt $90045^{\circ} 39$ '50.70”N $11^{\circ} 08^{\prime} 12.93$ ”E 6.IV.2012 leg. Sette - Parricelli 15 Ex.; Val Fraselle mt 900 Vaglio sotto conifera

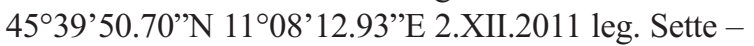
Parricelli 3 Ex.; Val Revolto mt $105045^{\circ} 40^{\prime} 56.02^{\prime \prime} \mathrm{N}$ $11^{\circ} 07^{\prime} 01.57^{\prime \prime}$ E 14.XI.2011 leg. Sette 3 Ex.; Val Revolto

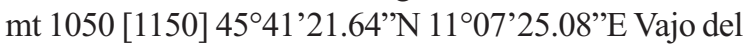
Diavolo 18.IV.2012 leg. Sette 10 Ex. Aed. Präp.; Val Revolto mt 1200 Rifugio Boschetto 4541'25.74'N $11^{\circ} 07^{\prime} 13.53$ 'E 5.VIII.2011 leg. Sette 1 Ex.; Val Revolto mt 900 Primo tornante - Rive del progno Lavaggio del 


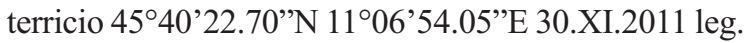
Sette 1 Ex.; Val Revolto mt 900 Primo tornante Rive del

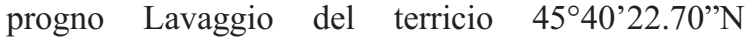

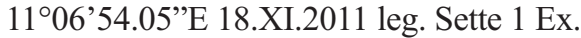

Außerhalb Parco Lessinia: Val Tramigna (VR) Loc. Sengelle [Cengelle] mt $300 \quad 45^{\circ} 30^{\prime} 03.19^{\prime \prime} \mathrm{N}$ $11^{\circ} 11^{\prime}$ '52.19'E 19.I.2010 leg. Sette 1 Ex. Aed. Präp., $45^{\circ} 30^{\prime} 03.19^{\prime \prime} \mathrm{N} \quad 11^{\circ} 11^{\prime} 52.19^{\prime \prime} \mathrm{E}$ 9.I.2010 1 Ex., $45^{\circ} 30^{\prime} 02.53^{\prime \prime} \mathrm{N} 11^{\circ} 12^{\prime} 20.87^{\prime \prime} \mathrm{E}$ 5.XI.2009 1 Ex.

BESCHREIBUNG. Körperlänge 0,97-1,08 mm. Einfarbig hell rotbraun gefärbt.

Fühler mit 3-gliedriger Keule. Glieder VI und VIII kleiner als die benachbarten, II 1,5 mal so lang wie breit, der Länge nach deutlich gerundet, III - VI zunehmend rundlicher, VII Übergangsglied zwischen den vorhergehenden rundlichen und den nachfolgenden krugförmigen VIII, IX, X. XI kurzoval.

Länge: Breite der Fühlerglieder II 9:6, III 5:5, IV 5:5, V 6:6, VI 4,5:5,5, VII 6:6,5, VIII 5:6,5, IX 7:9, X 9:11, XI 16:12,5.

Pronotum: Etwa 1,2 mal so breit wie lang. GröBte Breite des Pronotums 0,38-0,43 mm, wenig vor der Längsmitte des Pronotums. Seitenränder nach vorn gleichmäßig gerundet stark konvergierend, nach hinten geradlinig schwach konvergierend. Seitenränder jeweils mit ca. 11 Porenpunkten, aus denen nach hinten gerichtete Setae entspringen. Durch die Porenpunkte sind die Seitenränder gekerbt. Hinterwinkel abgerundet und konkav gegen den Seitenrand abgesetzt. Vor den Hinterecken vom Hinterrand und vom Seitenrand etwa gleich weit entfernt mit einem kleinen Grübchen. Ohne mediane Beule vor dem Hinterrand. Punktierung des Pronotums wie der Elytren mäßig stark. Pubeszenz auf Pronotum und Elytren wenig abstehend, nach hinten gerichtet, auf den Elytren ca. 1/3 länger als auf dem Pronotum.

Elytren: Etwa 1,42 mal so lang wie zusammen breit. Hinterrand gerade abgestutzt. Basale Pore nur von einem Büschel aus nach hinten gerichteten, feinen Setae überdeckt. Feine Setae nur in einem kleinen Feld vorhanden, das sich nach vorn und außen an die Pore anschließt.

Metasternum: Beiderseits der Mittellinie in einem breiten Längsstreifen zwischen den Mesocoxae und den Metacoxae dicht und grob punktiert, dazwischen und seitlich davon viel feiner und weitläufiger. Kielchen des Metasternalfortsatzes kurz und nicht verlängert.
Sexualdimorphismus: Metasternum beim $\widehat{\partial}$ median breit flach vertieft, beim $q$ median abgeflacht und nicht vertieft. Protibien im apikalen Drittel bei $\widehat{\sigma}$ und $q$ gleichartig innen wenig abgeflacht. Aedeagus (Abb. 1M).

Derivatio nOMinis. Der Name (Adjektiv) bezieht sich auf das stark punktierte Metasternum.

Vergleich. Von dem sehr ähnlichen Cephennium (Neocephennium) fulvum Schaum, 1859 durch die Gestalt der ausstülpbaren Sklerite des Aedeagus und die Punktierung des Metasternums verschieden.

Bionomie. Die Exemplare wurden aus der Streu von Misch-, Nadel-, Kastanien-, Buchen-Wäldern, an Bachufern, auf kalkreichem Boden, oft im Tal, in Höhenlagen zwischen 300 und 1750 m gesammelt.

\section{ANMERKUNG}

Ein Weibchen vom Monte Baldo ("sopra Brenzone mt 600 10.IX.1979 leg. A.Sette") gehört in das Subgenus Geodytes Saulcy, 1864. Ohne ein Männchen lässt es sich nicht weiter zuordnen. Das Subgenus Geodytes ist in der westlichen Mediterraneis mit vielen, ganz überwiegend unbeschriebenen Arten vertreten. Am Südrand der Alpen sind bisher nur Vorkommen westlich des Gardasees bekannt. Damit ist der Fund einer GeodytesArt auf der Ostseite des Gardasees bemerkenswert.

TABELLARISCHE ÜBERSICHT ZUR UNTERSCHEIDUNG der Arten der Untergattung Cephennium der Provinz Verona

Die Arten der Gattung Cephennium sind ohne Untersuchung des Aedeagus nicht sicher zu determinieren, es sei denn, alle Arten eines bestimmten Gebiets sind bekannt. Dies dürfte für die Provinz Verona zutreffen (Tab. 1). Zwar ist es dann immer noch am einfachsten, den Aedeagus zur Determination zu verwenden. Die Männchen der Arten lassen sich dann leicht bestimmen. Doch lassen sie sich auch ohne die dafür nötige Präparation voneinander unterscheiden. Besonders hilfreich ist dabei eine Untersuchung der Protibien und des Metasternums. Die Fühlerglieder sind zur Artunterscheidung kaum geeignet. Sie sind im Querschnitt nicht kreisrund, so dass ihre Proportionen allein durch eine unterschiedliche Lage am präparierten Exemplar voneinander abzuweichen 
Tab 1. Übersicht zur Unterscheidung der Arten der Untergattung Cephennium der Provinz Verona.

\begin{tabular}{lllll}
\hline & lessinicum & rossii & settei & jureceki \\
\hline Körperlänge in mm & $0,92-1,02$ & $1,00-1,08$ & $1,07-1,24$ & $1,19-1,36$ \\
Breite des Pronotums in mm & $0,40-0,44$ & $0,44-0,46$ & $0,46-0,50$ & $0,50-0,58$ \\
Beule des Pronotums & Schwach & Fehlt & Kräftig & Kräftig \\
\multirow{\jmath}{*}{ Einbuchtung der Protibia } & Minimal & Mittel & Mittel & Stark \\
\multirow{2}{*}{ Eindruck des Metasternums } & Schwach & Tief & Tief & Tief \\
Punktierung des Metasternums & Fein & Fein & Kräftig seitlich der Mitte & Kräftig nur vor den \\
& & & & Metacoxen \\
Hinterrand der Elytren abgestutzt & Wenig & Breit & Wenig & Breit \\
\hline
\end{tabular}

scheinen. Die Determination einzelner Weibchen bleibt eventuell unsicher.

Typische Exemplare aus der Provinz Verona lassen sich schon allein nach der Körpergröße zuordnen.

Die Tabelle ist nicht auf solche Exemplare von Cephennium rossii anzuwenden, die aus anderen Regionen stammen (s.o.).

\section{DANKSAGUNG}

Die Aufsammlungen von Alberto Sette in der Provinz Verona und Kopien der Zeichnungen, die Claude Besuchet von Aedeagi der Cephennium-Arten angefertigt hat, standen für die Publikation zur Verfügung. Dafür sei Alberto Sette und Claude Besuchet herzlich gedankt.

\section{LITERATUR}

Castellini G., 2011 - Cinque nuove specie italiane di Cephennium (Coleoptera Scydmaenidae). Bollettino della Società Entomologica Italiana, 143(2): 77-84.

MachulKa V., 1930 - Eine vergleichende Studie über das Cephennium montanum Rttr. und ihm ähnliche Arten aus Nord-Italien. Wiener Entomologische Zeitung, 47: 11-18. 